\title{
Improved Description of Bremsstrahlung for High-Energy Electrons in Geant4
}

\author{
Andreas Schälicke, Vladimir Ivanchenko, Michel Maire, Laszlo Urban
}

\begin{abstract}
Geant4 [1] provides a software toolkit for the simulation of particles through matter. It has wide range of applicability, ranging from high-energy particle physics to space science.

The physics description of Geant 4 is in a constant process of improvement and validation. In this work a new relativistic Bremsstrahlung model is introduced. The key feature is a consistent description of matter effects, esp. the Landau-PomeranchukMigdal (LPM) effect. The LPM effect will be of paramount importance for experiments at modern high-energy colliders, esp. the LHC, as well as in astroparticle physics applications.
\end{abstract}

Index Terms-Bremsstrahlung, electromagnetic interactions, particle interactions, physics validation, simulation.

\section{INTRODUCTION}

B REMSSTRAHLUNG is the dominant electromagnetic process for high-energy electron beams interacting with matter. The cross section is well known [2], [3], and the theoretical knowledge has been continuously improved over the years [4]-[7]. But for a good description of modern experiments also the correct incorporation of media effects plays an important role. A prominent example is the LandauPomeranchuk-Migdal (LPM) effect. It has been predicted already some time ago [8], but only in the past decade it was verified by experiment [9], [10].

\section{THE LANDAU-POMERANCHUK-MIGDAL EFFECT}

It is a common feature of electromagnetic interactions at very high energies that the process of creation of particles is spread over a finite region of space. Its longitudinal dimension is called the formation length. For instance the LPM effect occurs, when a high-energetic charged particle traverses through dense matter, then the formation length of bremsstrahlung radiation is of the order of the wave length of the emitted photon. When the formation length exceeds the typical scale where multiple scattering occurs, the photon emission probability is suppressed. The size of the suppression depends on the environment. The combination of different material effects, in particular the Ter-Mikaelian effect [11] and the Landau-Pomeranchuk-Migdal effect, needs special attention and is presented in this work.

A. Schälicke is with DESY, 15738 Zeuthen, Germany, (e-mail: andreas.schaelicke@desy.de).

V. Ivanchenko is with CERN, Geneva, CH-1211, Switzerland, on leave of Ecoanalytica, Moscow State University, 119899 Moscow, Russia.

M. Maire is with Institut National de Physique Nucleaire et de Physique des Particules (LAPP), Annecy-le-vieux 74941, France.

L. Urban is with Research Institute for Particle and Nuclear Physics (RMKI), Budapest H-1525, Hungary.

\section{A RELATIVISTIC BREMSSTRAHLUNG MODEL}

The basis of the implementation is the well known high energy limit of the Bremsstrahlung process [5],

$$
\begin{aligned}
& \frac{d \sigma}{d k}=\frac{4 \alpha r_{e}^{2}}{3 k}\left[\left\{y^{2}+2\left[1+(1-y)^{2}\right]\right\}\right. {\left[Z^{2}\left(F_{\text {el }}-f\right)+Z F_{\text {inel }}\right] } \\
&\left.+(1-y) \frac{Z^{2}+Z}{3}\right]
\end{aligned}
$$

The elastic from factor $F_{\text {el }}$ and inelastic form factor $F_{\text {inel }}$, describe the scattering on the nucleus and on the shell electrons, respectively, and for $Z>4$ are given by [12]

$$
F_{e l}=\log \left(\frac{184.15}{Z^{1 / 3}}\right) \quad \text { and } \quad F_{\text {inel }}=\log \left(\frac{1194 .}{Z^{2 / 3}}\right) .
$$

This corresponds to the complete screening approximation. The Coulomb correction is given by [12]

$$
f=\alpha^{2} Z^{2} \sum_{n=1}^{\infty} \frac{1}{n\left(n^{2}+\alpha^{2} Z^{2}\right)}
$$

This approach provides an analytic differential cross section for an efficient evaluation in a Monte Carlo computer code. Note that in this approximation the differential cross section $d \sigma / d k$ is independent of the energy of the initial electron.

The total integrated cross section $\int d \sigma / d k d k$ is divergent, but the energy loss integral $\int k d \sigma / d k d k$ is finite. This allows the separation of the simulation treatment in two parts. The first part describes the discrete production of photons with a minimal energy $E_{c u t}$ and is given by

$$
\sigma(E)=\int_{E_{\text {cut }}}^{E_{\max }} \frac{d \sigma}{d k} d k
$$

The second part governs continuos energy loss

$$
\frac{d E}{d x} \sim \int_{0}^{E_{\text {cut }}} k \frac{d \sigma}{d k} d k
$$

This is a standard treatment in Geant4 electromagnetic physics processes. The minimal energy value $E_{c u t}$ depends on the problem domain and has to be set by the user. ${ }^{1}$ The result can be compared with more sophisticated cross section evaluation [4], which shows excellent agreement provided the energy of the initial electron exceeds $1 \mathrm{GeV}$.

At higher energies, on the other hand, matter effects become more and more important. In this work we consider the two

${ }^{1}$ Geant 4 provides a method to infer the energy cut from a cut in particle range. 
leading effects, the LPM effect and the Ter-Mikaelian effect. The analytic cross section representation, eq. (1), provides an ideal basis for the incorporation of these matter effects.

The first effect is the Landau-Pomeranchuk-Migdal effect. We choose the evaluation based on [6], [8], [13]

$$
\begin{aligned}
\frac{d \sigma}{d k}= & \frac{4 \alpha r_{e}^{2}}{3 k}\left[\xi(s)\left\{y^{2} G(s)+2\left[1+(1-y)^{2}\right] \phi(s)\right\}\right. \\
& \left.\times\left[Z^{2}\left(F_{e l}-f\right)+Z F_{\text {inel }}\right]+(1-y) \frac{Z^{2}+Z}{3}\right]
\end{aligned}
$$

The LPM suppression functions are defined by [8]

$$
G(s)=24 s^{2}\left(\frac{\pi}{2}-\int_{0}^{\infty} e^{-s t} \frac{\sin (s t)}{\sinh (t / 2)} d t\right)
$$

and

$$
\phi(s)=12 s^{2}\left(-\frac{\pi}{2}+\int_{0}^{\infty} e^{-s t} \sin (s t) \sinh (t / 2) d t\right)
$$

They can be piecewise approximated with simple analytic functions, see e.g. [13]. The suppression function $\xi(s)$ is recursively defined via

$$
s=\sqrt{\frac{k E_{\mathrm{LPM}}}{8 E(E-k) \xi(s)}}
$$

but can be well approximated using an algorithm introduced by [13].

We define the material dependent parameter $E_{\mathrm{LPM}}$ to be

$$
E_{\mathrm{LPM}}=X_{0} \frac{\alpha m_{e}^{2}}{8 \pi \hbar c}
$$

according to [9]. Note that this definition differs from other definition (e.g. [6]) by a factor $1 / 2$.

An additional multiplicative factor governs the suppression via density effect (Ter-Mikaelian effect).

$$
S(k)=\frac{k^{2}}{k^{2}+k_{p}^{2}}
$$

The characteristic photon energy scale $k_{p}$ is given by the plasma frequency of the media, defined as

$$
k_{p}=\hbar \omega_{p} \frac{E_{e}}{m_{e} c^{2}}=\frac{\hbar E_{e}}{m_{e} c^{2}} \cdot \sqrt{\frac{n_{e} e^{2}}{\epsilon_{0} m_{e}}} .
$$

Particular emphasis has been put the correct treatment of overlap region, where both suppression mechanism, LPM and Ter-Mikaelian, are present. Here we follow a procedure suggested by [14]. The basic idea is to turn off LPM suppression as the density effect takes in. This could be achieved by defining a modified suppression variable $\hat{s}$ via

$$
\hat{s}=s \cdot\left(1+\frac{k_{p}^{2}}{k^{2}}\right)
$$

and using $\hat{s}$ in the LPM suppression functions instead of $s$.

The presented algorithms could be translated into Monte Carlo code, which proved to perform efficiently for moderately high energies ( $1 \mathrm{GeV}$ to $100 \mathrm{TeV})$. Details will be presented elsewhere, [15].

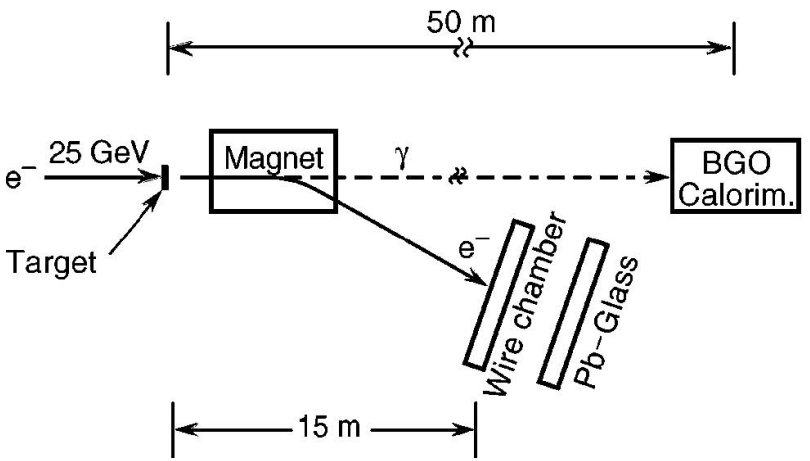

Fig. 1. The Layout of the E-146 experiment at SLAC, more details can be found in [9].

\section{VALIDATION}

In the standard implementation of Bremsstrahlung in Geant4 the simulation is based on a parameterization of theory [4] as well as on experimental data [16]. In recent years some validation of electromagnetic processes in Geant 4 has been performed at small and intermediate energies [17]. In this work Bremsstrahlung description for very high energetic electrons is analyzed.

We confront the results of a simulation, including the new implementation of the Bremsstrahlung process, with data from recent experiments at the SPS at CERN [10], and at SLAC [9]. The experiments differ in the choice of beam energy and materials, as well as in the range of the observed Bremsstrahlung photon spectrum.

The SLAC experiment E-146 was dedicated to the quantitative validation of the LPM and Ter-Mikaelian effect. The setup is shown in figure 1 . The experiment was performed with electron beam energies $8 \mathrm{GeV}$ and $25 \mathrm{GeV}$, and with different target materials in varying thicknesses. The photon energy was measured in the interval from $200 \mathrm{keV}$ to 500 $\mathrm{MeV}$. Some selected results are shown in figures 2 to 4 . Note that even for targets as thin as a few percent of a radiation length, the probability to radiate more than one photon is not negligible, and has to be taken into account in the simulation.

The solid line in plots 2 to 4 represents the Geant4 simulation using the new model including LPM and TerMikaelian effect. For comparison simulation results without LPM effect are also shown as dashed lines. For light materials like Calcium, Fig. 2 the Ter-Mikaelian effect is dominant. For heavier materials, e.g. Uranium in Fig. 3, the LPM effect becomes visible, even for energies as small as $8 \mathrm{GeV}$. Figure 4 gives a nice example of the transition between Ter-Mikaelian and LPM effect. Note that in Fig. 3 and 4 the simulation results have been scaled up by $20 \%$ in order to meet the data.

In general the agreement with data is reasonalbly good. According to the authors of the experiment, the deviation at very small photon energies and high electron beam energy is likely caused by transition radiation photons and residual background.

Recently another experiment dedicated to the LPM effect in Bremsstrahlung process for multihundred $\mathrm{GeV}$ electrons has been carried out at the CERN SPS. The experimental setup is 

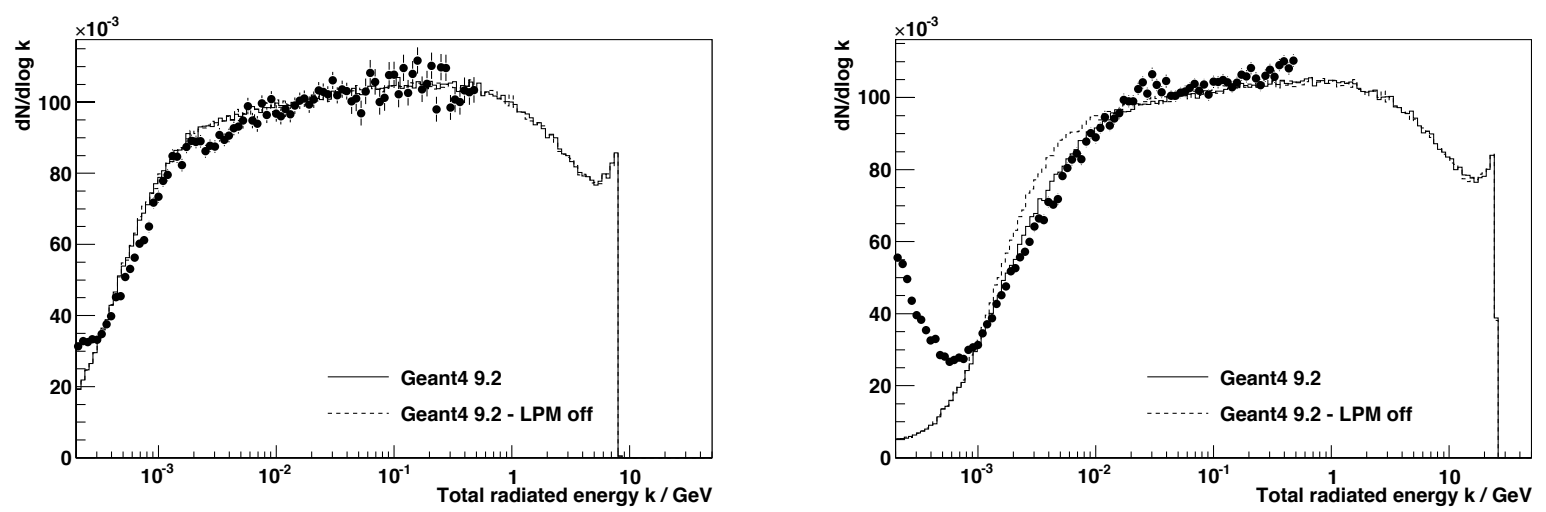

Fig. 2. Bremsstrahlung spectrum for an $8 \mathrm{GeV}$ (a) and $25 \mathrm{GeV}$ (b) electron beam incident on a $4.1 \mathrm{~mm}\left(2 \% X_{0}\right)$ Calcium target. Solid lines show Geant 4 simulation results with Ter-Mikaelian and LPM effect included. Dashed lines correspond to a simulation without LPM effect. The data are taken from [9].
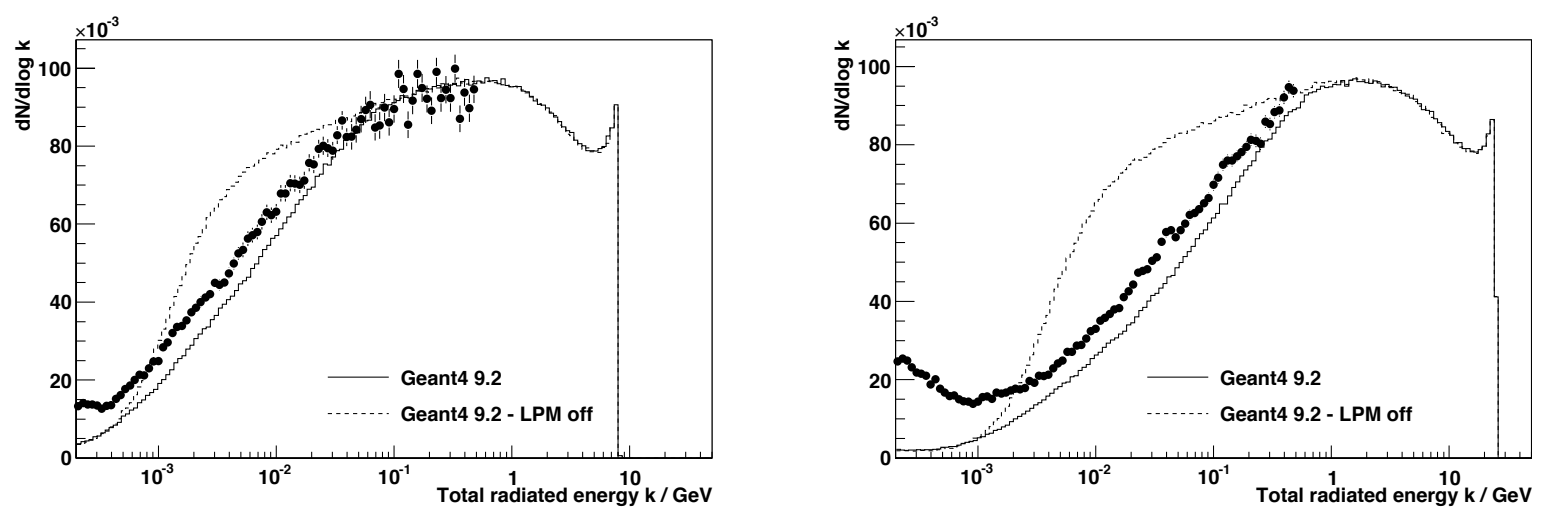

Fig. 3. Bremsstrahlung spectrum as in Fig. 2 but for an $8 \mathrm{GeV}$ (a) and $25 \mathrm{GeV}$ (b) electron beam incident on a $0.15 \mathrm{~mm}\left(5 \% X_{0}\right) \mathrm{Uranium}$ target.

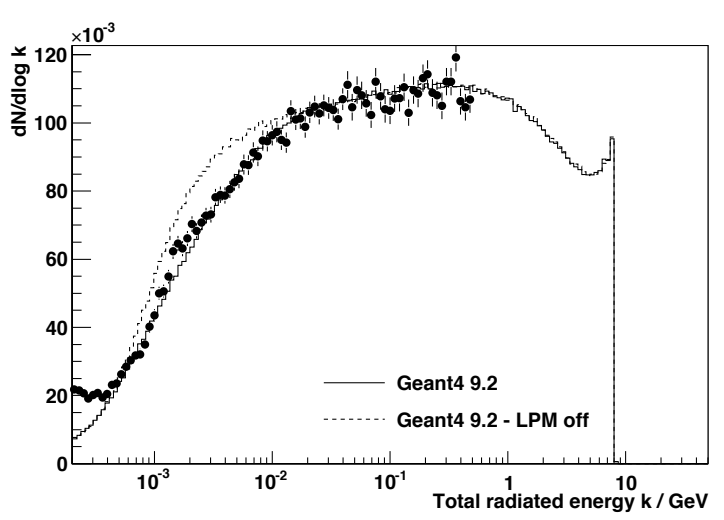

Fig. 4. Bremsstrahlung spectrum as in Fig. 2 but for an $8 \mathrm{GeV}$ electron beam incident on a $0.5 \mathrm{~mm}\left(3 \% X_{0}\right)$ Iron target.

similar to the SLAC experiment. The electron energies of 149 $\mathrm{GeV}, 207 \mathrm{GeV}$ and $287 \mathrm{GeV}$ have been studied. The target materials investigated are Copper, Tantalum and Iridium. The photon spectrum has been measured starting with $2 \mathrm{GeV}$.
Corresponding to the higher electron energies, the impact of the LPM effect is much stronger in this experiment. Fig. 5 shows results for an $207 \mathrm{GeV}$ electron beam incident on an Iridium target. Two other examples are given in Fig. 6 and in Fig. 6. Again the Geant4 simulation with the new relativistic model (solid line) shows good agreement with the data. Also shown are results as expected without LPM effect (dashed line), and for comparison also results of a previous, more simple implementation of the LPM effect in Geant 4 (red/grey solid line). This illustrates the impovement in the LPM description.

\section{CONCLUSION}

A new relativistic model for the description of high-energy electron Bremsstrahlung has been introduced in the Geant4 software framework. It is applicable for energies above a few $\mathrm{GeV}$. The key feature is the consistent incorporation of the LPM and the Ter-Mikaelian effect. The comparison of simulation results with data of dedicated experiments at CERN and SLAC show in general good agreement. The new model provides an improved description of very high energy particle interactions with mater, both for high-energy physics and astroparticle physics applications. 


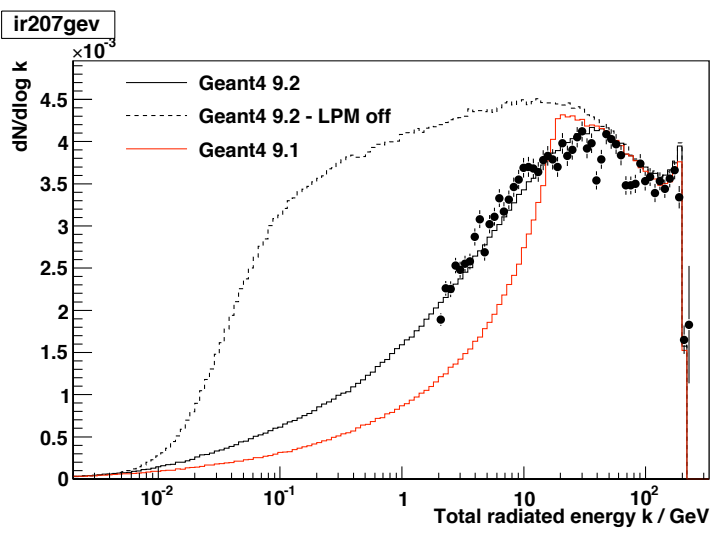

Fig. 5. Bremsstrahlung spectrum for $207 \mathrm{GeV}$ electrons on a $0.128 \mathrm{~mm}(4 \%$ $X_{0}$ ) Iridium target. The dotted line is the result of Geant 4 simulation without LPM effect, the full line includes the LPM suppression. For comparison results of a previous Geant 4 release (red/grey solid line) are also shown. The data are taken from [10].

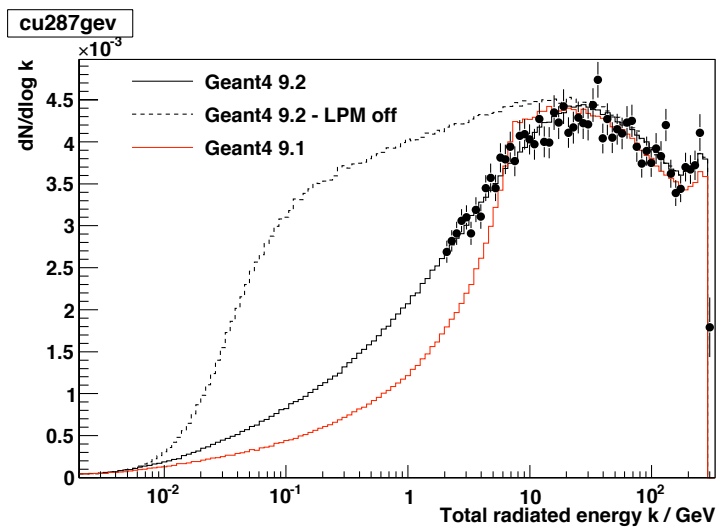

Fig. 6. Bremsstrahlung spectrum as in Fig. 5 but for $287 \mathrm{GeV}$ electrons on a $0.63 \mathrm{~mm}\left(4 \% X_{0}\right)$ Copper target.

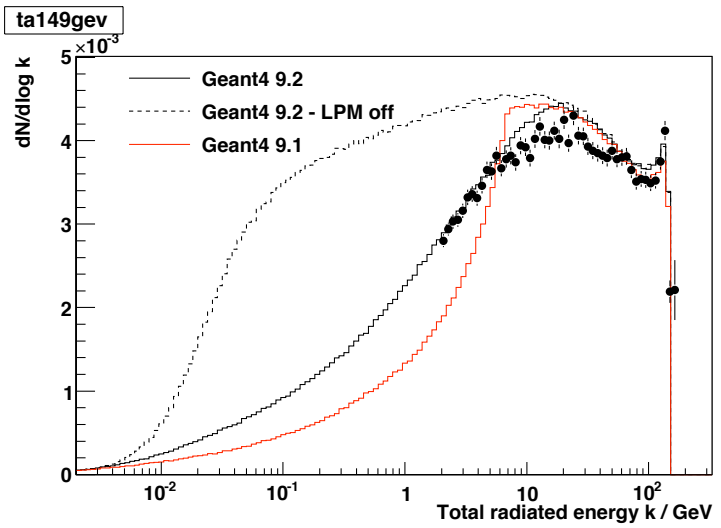

Fig. 7. Bremsstrahlung spectrum as in Fig. 5 but for $149 \mathrm{GeV}$ electrons on a $0.182 \mathrm{~mm}\left(4 \% X_{0}\right)$ Tantalum target.

It will be part of the upcoming Geant4 release 9.2 (December 2008).

\section{ACKNOWLEDGMENT}

Many thanks go to Spencer Klein and Ulrik I. Uggerhøj for help with the experimental data.

\section{REFERENCES}

[1] S. Agostinelli et al. (GEANT4 Collaboration), GEANT4: A simulation toolkit, Nucl. Instr. and Meth. A 506, 250 (2003); J. Allison et al., Geant4 developments and applications, IEEE Trans. Nucl. Sci. 53, 270 (2006).

[2] H.A. Bethe and W. Heitler, On the stopping of fast particles and the creation of positive electrons, Proc. R. Soc. London, A 146 (1934) 83.

[3] Y-S. Tsai, Pair production and bremsstrahlung of charged leptons, Rev. Mod. Phys. 46 (1974) 815; Rev. Mod. Phys. 49 (1977) 421.

[4] S.M. Seltzer and M.J. Berger, Bremsstrahlung spectra from electron interactions with screened atomic nuclei and orbital electrons. Nucl. Inst. Meth. 12 (1985) 95.

[5] M.L. Perl, Notes on the Landau Pomeranchuk Migdal effect: experiment and theory, in Procede Les Rencontres de physique de la Valle D'Aoste, SLAC-PUB-6514.

[6] S. Klein, Suppression of bremsstrahlung and pair production due to environmental factors, Rev. Mod. Phys. 71 (1999) 1501-1538.

[7] V.N. Baier, B.M. Katov, Concept of formation length in radiation theory, Phys. Rep. 409 (2005) 261.

[8] A.B. Migdal, Bremsstrahlung and Pair Production in Condensed Media at High Energies, Phys. Rev. 103 (1956) 1811.

[9] P.L. Anthony et al., Bremsstrahlung suppression due to the LandauPomeranchuk-Migdal and dielectric effects in a variety of materials, Phys. Rev. D 56 (1997) 1373, SLAC-PUB-7413/LBNL-40054.

[10] H.D. Hansen et al., Landau-Pomeranchuk-Migdal effect for multihundred GeV electrons, Phys. Rev. D 69 (2004) 032001.

[11] M.L. Ter-Mikaelian, Dokl. Akad. Nauk SSSR 94 (1954) 1033.

[12] C. Amsler et al.,2008 Review of Particle Physics, Physics Letters B667, 1 (2008).

[13] T. Stanev et.al., Development of ultrahigh-energy electromagnetic cascades in water and lead including the Landau-Pomeranchuk-Migdal effect Phys. Rev. D25 (1982) 1291.

[14] M.L.Ter-Mikaelian, High-energy Electromagnetic Processes in Condensed Media, Wiley, 1972.

[15] A. Schälicke et al., in preparation.

[16] S.T. Perkins, D.E. Cullen, S.M. Seltzer, Evaluated Electrons Data Library, UCRL-50400 Vol.31.

[17] S. Chauvie et al., Validation of the Geant4 bremsstrahlung models, IEEE NSS Conference Record, Volume 3, (2006) 1511. 